\title{
Reflets
}

Revue ontaroise d'intervention sociale et communautaire

\section{Sortir la spiritualité du placard}

\section{Diane Dupont}

Volume 12, numéro 1, 2006

Spiritualité et intervention sociale

URI : https://id.erudit.org/iderudit/013443ar

DOI : https://doi.org/10.7202/013443ar

Aller au sommaire du numéro

Éditeur(s)

Reflets : Revue ontaroise d'intervention sociale et communautaire

ISSN

1203-4576 (imprimé)

1712-8498 (numérique)

Découvrir la revue

Citer cet article

Dupont, D. (2006). Sortir la spiritualité du placard. Reflets, 12(1), 168-180.

https://doi.org/10.7202/013443ar

Tous droits réservés (C) Reflets : Revue ontaroise d'intervention sociale et communautaire, 2006

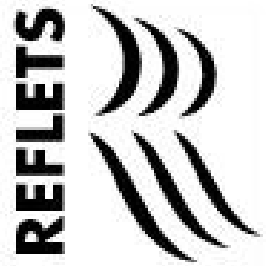

Ce document est protégé par la loi sur le droit d'auteur. L'utilisation des services d'Érudit (y compris la reproduction) est assujettie à sa politique d'utilisation que vous pouvez consulter en ligne.

https://apropos.erudit.org/fr/usagers/politique-dutilisation/ 


\section{Sortir la spiritualité du placard}

"...dans les

professions d'aide, les manifestations spirituelles et religieuses ont souvent été abordées sous l'angle de pathologies de personnes considérées comme " anormales ", mettant ainsi de côté leur contribution en tant que forces et ressources profitant à la personne aidée. »

\section{Diane Dupont}

Je me réjouis du fait qu'un numéro de Reflets soit consacré à la spiritualité en travail social. Il y a à peine une quinzaine d'années, aucune faculté de sciences sociales n'aurait osé aborder ce thème. Longtemps, les professionnels de l'intervention sociale ont évité la question. Pourtant, en Amérique du Nord, les origines du travail social sont empreintes d'une spiritualité ayant inspiré de nombreuses communautés religieuses à mettre en place des structures visant à répondre aux besoins sociaux de l'époque.

Deux facteurs ont contribué à évacuer la spiritualité des œuvres caritatives à leur origine. Le premier est la séparation progressive de l'Église et de l'État et le deuxième, la "laïcisation » des œuvres caritatives, allant de pair avec la professionnalisation du travail social. Celui-ci devait alors se bâtir des assises professionnelles et démontrer sa nature scientifique, au même titre que la médecine et la psychologie. La révolution scientifique avait misé sur le démontrable, l'analyse, l'objectivité. Conséquemment, on se détacha de toute forme de spiritualité, laquelle était perçue comme non scientifique et trop subjective, parce que fondée sur l'expérience et l'intuition. De plus, dans les professions d'aide, les manifestations spirituelles et religieuses ont souvent été abordées sous l'angle de pathologies de personnes considérées comme " anormales ", mettant ainsi de côté leur contribution en tant que forces et ressources profitant à la personne aidée.

Enfin, plus que toute autre discipline, le travail social est exposé à l'impact qu'ont le pouvoir et l'oppression sur la vie des individus. De ce fait, elle évite tout cadre de référence qui pourrait être perçu comme un schème imposé par l'intervenante 
"La remise en question des approches en travail social que cette conjoncture provoque nous invite à envisager d'autres perspectives pour nous laisser interpeller, ouvrir notre espace de réflexion à des idées nouvelles, parfois même déroutantes. » ou l'intervenant social à la personne en état de vulnérabilité parce qu'en situation de crise.

Avec l'essor de la pensée postmoderne, le questionnement sur la place de la spiritualité dans les sciences sociales a refait surface comme facette qu'il importe de considérer dans la relation d'aide. Au Canada français, nous n'en sommes qu'à nos premiers balbutiements dans cette réflexion sur le rôle et l'apport de la spiritualité dans la relation d'aide. Personnellement, je considère comme très enrichissants et positifs ce questionnement et ce débat sur le rôle de la spiritualité dans le cadre du travail social. La remise en question des approches en travail social que cette conjoncture provoque nous invite à envisager d'autres perspectives pour nous laisser interpeller, ouvrir notre espace de réflexion à des idées nouvelles, parfois même déroutantes. Cela nous amène également à réfléchir sur notre propre spiritualité dans la vie quotidienne, tant dans notre espace d'intimité que dans notre vie professionnelle.

Dans les pages suivantes, je me permets de sortir des sentiers battus de l'objectivité des sciences sociales pour explorer quelques pistes sur la spiritualité, son impact sur les intervenants et les intervenantes en travail social et son utilisation dans la relation d'aide. Mes propos sont teintés de mon expérience en tant que Canadienne française d'origine, ayant grandi dans la religion catholique, cheminé spirituellement dans la foi chrétienne, œuvré pendant plusieurs années dans le contexte de travail et d'intervention féministes, travaillé dans des contextes multiculturels et côtoyé des personnes qui ont adhéré à diverses croyances, religions, pratiques spirituelles et rituelles autres que les miennes. Toutes ces rencontres et ces circonstances ont nourri et continuent d'alimenter ma réflexion et de m'interpeller quotidiennement. En fait, chaque rencontre avec une personne, que ce soit dans la vie personnelle ou dans la relation d'aide dans un contexte de travail, est un avènement qui vient enrichir mon cheminement spirituel-humain parce qu'elle m'ouvre la possibilité de découvrir et d'explorer une nouvelle facette de la vie. 


\section{Quelques pistes sur la spiritualité}

\section{La spiritualité comme quête de sens}

"La quête spirituelle est une belle aventure d'humanité."

\author{
"L'être humain n'est jamais purement être : il impli- \\ que toujours une signification." (A. Heschel)
}

Chaque jour, l'être humain reprend sa quête à « savoir si la vie mérite, ou non, d'être vécue » (A. Camus). C'est ce sens dernier, ce sens global, que l'on veut attribuer à la vie. Ce choix fondamental unifie ensuite toutes les actions et leur donne une direction, un sens.

Toutefois, cette longue quête de sens n'est jamais terminée. Bien au contraire. Chaque fois que la personne croit avoir levé le voile sur le sens de son existence, elle se rend vite compte qu'elle s'est plongée encore plus profondément dans l'énigme de son existence et de celle qui l'entoure. Le théologien Jürgen Moltmann affirme que cet éternel questionnement sur soi, en tant qu'être humain, maintient l'humanité vivante et ouverte. La quête spirituelle est une belle aventure d'humanité :

S'il ['être humain] est en même temps le questionneur et le questionné, il est inévitable que toutes les réponses qu'il se donne ou qu'il se fait donner par d'autres soient insuffisantes et posent pour lui un nouveau point d'interrogation. De même qu'il essaie d'aller derrière les choses pour les connaître et les utiliser, il voudrait en somme aller aussi derrière lui-même pour se connaître. Mais parce que c'est lui-même qui veut aller derrière lui-même, l'objet de sa recherche lui glisse toujours des doigts et, à mesure que s'offrent à lui de plus nombreuses possibilités de solution sous la forme d'esquisses de l'être humain, il devient de plus en plus une énigme pour lui-même. Plus le nombre des réponses possibles s'élève, plus il a l'impression de se trouver dans une salle pleine de miroirs et de masques, et plus il devient obscur à ses propres yeux. 
"Ainsi, la spiritualité est la prise de conscience que la réalité est quelque chose de beaucoup plus vaste que l'expérience que nous en faisons. "
Aussi l'être humain est en fait pour l'être humain le plus grand des mystères. Il doit se connaître pour vivre et pour se rendre connaissable aux autres. Mais il doit en même temps rester caché à lui-même pour demeurer en vie et dans la liberté. Si, en effet, il parvenait enfin "derrière lui-même ", il pourrait constater ce qui ne va pas chez lui, mais alors plus rien n'irait mal pour lui, tout serait rigoureusement fixé, et il serait au terme. L'"énigme résolue " de l'être humain serait en même temps la liquidation de l'humanité. Dans la mesure où nous connaissons l'être humain, nous le connaissons comme interrogation, comme liberté et ouverture. ${ }^{1}$

Ainsi, la spiritualité est la prise de conscience que la réalité est quelque chose de beaucoup plus vaste que l'expérience que nous en faisons. Cette "réalité-beaucoup-plus-vaste-que-nosexpériences " entraine une transformation et une expansion de notre conscience et une ouverture pour explorer l'inconnu. Ce processus d'entrer en relation avec cette réalité constitue l'expérience spirituelle.

Le cheminement spirituel n'est pas une entité statique qui nous ferait stagner dans une réponse facile aux grands questionnements de l'existence. Il n'est pas, non plus, un parcours linéaire qui nous transporte en ligne droite du point A au point B. La spiritualité d'un individu se bâtit au fil d'événements et d'expériences qui apportent une nouvelle perspective, ou dimension, spirituelle. L'illumination acquise hier n'aura pas nécessairement la même signification et la même résonance aujourd'hui. La perception du sens de la vie qu'une personne avait à 20 ans ne sera pas nécessairement la même lorsqu'elle aura 40 ans, 50 ans, 60 ans... Les événements lui apporteront de nouveaux questionnements et bouleverseront son schème de croyances et sa façon de comprendre la vie.

\section{La spiritualité en mouvement-action}

La spiritualité apporte une autre dimension à la connaissance, à l'intuition et à la sagesse : connaître en "étant ". La connaissance 
"Il faut donc reconnaître que spiritualité et engagement social ont besoin de s'alimenter l'un l'autre." n'est pas seulement l'illumination de l'esprit par les concepts et les idées, mais elle engage tout l'être à traduire cette connaissance dans ses activités quotidiennes; elle oriente ses choix de vie et ses engagements.

Le drame actuel de plusieurs sociétés occidentales ayant mis l'accent sur la consommation est que beaucoup de personnes se laissent bercer par les distractions extérieures et sont devenues étrangères à elles-mêmes. Sans minimiser la contribution des médias et des technologies qui ont fait de notre monde un grand village, nous devons nous poser la question à savoir si nous ne sommes pas devenus des actrices et des acteurs, engourdis par la haute stimulation d'informations transmises sur des écrans de toutes sortes, à attendre passivement le sens de nos existences. Ce que l'on appelle la "télé-réalité " n'est-il pas en train de nous déraciner du vrai terreau de la réalité qui se tisse dans la rencontre avec les autres, de nos apprentissages que nous faisons de nos expériences vécues? D'ailleurs, l'expérience existentielle est le contraire de la passivité. Son étymologie même renvoie à la «mise en mouvement, passage par, rupture d'avec, essai ou expérimentation, risque, itinéraire poussé jusqu'au bout, au-delà de quoi il n'y a rien ».2 La spiritualité nous incite donc à vivre notre vie le plus authentiquement possible, selon nos allégeances - ou non-allégeances - religieuses et nos croyances, dans le but de nous rapprocher de la réalité. Alors, notre vie trouve tout son sens dans ce processus de recherche. C'est dans le « ici et maintenant " que nos gestes quotidiens s'harmonisent à cet idéal qui s'est dessiné, et qui continue à se dessiner à partir de notre quête de sens. Il faut donc reconnaître que spiritualité et engagement social ont besoin de s'alimenter l'un l'autre. Une quête spirituelle qui exclurait toute forme d'engagement social risquerait de rester dans l'illusion. Par ailleurs, l'engagement social vide de cette quête risque de conduire l'humanité à la catastrophe. De même, une société qui fait la sourde oreille à ses « sages » risque de tourner en chaos qui compromettrait la dignité humaine. La spiritualité féministe et d'autres formes de spiritualité de libération soutiennent que l'activité politique et l'engagement social devraient faire partie de la spiritualité des individus. Que les 
"...l'être humain est finitude et pourtant, il ne s'épuise pas dans la finitude." forces économiques soient devenues si puissantes dans nos sociétés s'explique principalement par le fait que la spiritualité et l'éthique ont été évacuées de l'arène socio-politique et économique.

\section{La spiritualité devant la souffrance et la bêtise humaines}

La souffrance occasionnée par la maladie ou par des circonstances injustes engendre dans la plupart des cas une période de crise. Que nous la vivions dans notre personne ou que nous en soyons témoins dans la vie des autres, la souffrance fait tomber les sécurités que nous avions bâties par rapport à la vie et à notre quête de bonheur. Elle nous fait remettre en cause nos motivations et la signification que nous avions donnée à notre vie, à la vie en général, à nos convictions.

La souffrance nous place devant ce constat contradictoire : l'être humain est finitude et pourtant, il ne s'épuise pas dans la finitude. La crise provoquée par la souffrance stimule une nouvelle vision de vie. Il faut l'admettre, ce passage ne s'effectue pas sans heurts. Il nous faut lâcher prise par rapport aux valeurs, aux aspirations, à la compréhension que nous nous faisions de la réalité, ce que la tradition appelle "mourir à soi " pour passer à une autre compréhension de la réalité que nous avons à approfondir.

Il ne faudrait toutefois pas confondre ce lâcher-prise à une soumission passive aux injustices subies ou à toute forme de violence vécue. Le lâcher-prise est en fait l'aboutissement d'un long processus dans lequel la personne a :

- d'abord reconnu sa victimisation;

- accepté la victime en soi;

- confronté l'abuseur - soit directement, en personne, ou symboliquement dans le cadre de son processus de guérison;

- " relâché » la victime. Éventuellement, il faut relâcher la victime. Ce processus est à faire et à refaire, comme une spirale.Vouloir retenir cette facette de victime en nous, vouloir continuer à prendre soin et à bercer l'enfant blessé en soi pendant trop longtemps risque de ralentir et même d'arrêter le processus de croissance comme individus et comme société. Relâcher 
"Notre quête

spirituelle nous

amène à nous définir

en tant qu'être-en-

relation, avec soi, avec les autres et avec l'Autre. L'Autre étant toute entité qui nous dépasse, selon nos croyances ou nos convictions. ” la victime, lâcher prise requiert une force d'âme. Personne ne peut le faire à la place de l'autre, ni le forcer à le faire. Cette force libératrice émerge gracieusement de la personne qui est dans le processus de la guérison, quand elle est prête.

\section{La spiritualité comme moteur de nos relations}

Une des fonctions principales de la spiritualité est le décentrement de soi, plus précisément, en termes psychanalytiques, «l'expansion des frontières de l'ego".

Notre quête spirituelle nous amène à nous définir en tant qu'être-en-relation, avec soi, avec les autres et avec l'Autre. L'Autre étant toute entité qui nous dépasse, selon nos croyances ou nos convictions.

Après le rapport à la divinité, toutes les religions ont comme préoccupation principale d'établir les rapports entre les humains, de définir les relations entre eux. Bien que l'on puisse contester leur discours, force est d'admettre que la quête de l'être humain pour le divin suppose un mouvement vers l'autre, un engagement essentiel sur la voie de l'altérité.

L'être-en-relation implique nécessairement le dialogue, c'est-à-dire la réciprocité, l'échange où j'apprends à accueillir l'autre qui me révèle à moi-même. Cela nécessite une grande confiance en soi et en l'autre qui me permet de franchir les murs de résistance dressés par la peur et par le sentiment d'être menacé. Accueillir l'autre dans notre vie, c'est faire place au différent, à l'étranger. Tout être humain a pourtant besoin de l'hospitalité et de la compréhension des autres. Dans une société où la notion d'hospitalité est en train de disparaître, les êtres humains se sentent seuls. Alors, la notion d'empathie disparait parce que notre attention se fixe sur nous-mêmes et ne sait plus voir et reconnaitre l'autre ou encore, parce qu'une rupture s'est produite dans la confiance de l'un en l'autre. L'empathie, c'est l'habileté de reconnaître l'impact de la vie sur quelqu'un. 


\section{L'impact de la spiritualité en travail social}

L'analogie suivante nous présente le rôle de la travailleuse ou du travailleur social en lien avec la spiritualité dans la relation d'aide :

La vie humaine est comme une symphonie. Le corps est l'orchestre symphonique qui interprète et fait résonner cette symphonie. Ce genre musical obéit à une structure, la symphonie comptant plusieurs partitions, toutes reliées à l'intérieur de l'ouvre. Ainsi sont les dimensions de l'être humain qui ressemblent à ces partitions : appartenance sociale, engagement politique, développement psychologique, expérience spirituelle, etc. Toutes ces partitions sont essentielles à la réussite du chef-d'œuvre. Pendant l'exécution de l'œuvre, certains instruments restent en retrait pendant que d'autres sont mis en vedette. Mais, leur tour viendra au moment prévu par la structure symphonique. À d'autres moments, tous les instruments sont mis à contribution. Ce jeu de partitions qui s'entrecroisent fait toute la beauté de la symphonie.

Ainsi, peut-on comparer les événements que nous vivons à cette symphonie. Dans certains événements, ce sont certaines dimensions de l'être qui interviennent alors que les autres sont un peu plus en retrait, mais font toujours partie de la pièce musicale. Par exemple, certains événements contribueront ou auront surtout un impact sur le développement psychologique de l'individu alors que d'autres en appelleront davantage à ses ressources spirituelles. Puis, dans certains événements de sa vie, ce sont tous les éléments de son être qui interviendront en même temps.

Dans la relation d'aide, l'intervenante ou l'intervenant est comme le chef d'orchestre. Son rôle n'est pas de changer ou de réécrire la symphonie, mais bien de l'interpréter et s'assurer que les diverses partitions respecteront l'harmonie de l'œuvre et 
"D'où l'importance de s'accorder des espaces de réflexion, car le cheminement spirituel est un travail de longue haleine et nécessite des haltes permettant de faire le point et d'intégrer ce que nous apprenons de nos rencontres avec autrui. » l'inspiration de son créateur. Cela implique que le chef d'orchestre connait bien toutes les partitions musicales afin de les faire intervenir au moment opportun.

La spiritualité est donc l'une des partitions de la vie de la personne qui vient chercher de l'aide. L'intervenante ou l'intervenant ne pourrait ignorer cette partition, au risque de compromettre l'œuvre.

Indéniablement, l'intervenante ou l'intervenant ne pourrait ignorer la dimension spirituelle dans son intervention, sans toutefois la forcer ou l'imposer. Pour aborder l'aspect spirituel dans son intervention, l'intervenante ou l'intervenant doit connaitre un cheminement spirituel dans sa propre vie, non pas dans le but de dicter sa propre expérience à la personne aidée, mais plutôt pour ne pas se sentir menacé par le cheminement et les convictions spirituelles de cette dernière.

Dans un contexte de travail social, le cheminement spirituel de l'intervenante ou de l'intervenant implique une reconnaissance du caractère changeant de la vie, tant à l'intérieur qu'à l'extérieur de sa personne. La spiritualité est un élément dynamique. Les événements que nous vivons, les rencontres que nous faisons viennent parfois bouleverser, voire confronter, ce que nous tenions pour acquis. D'où l'importance de s'accorder des espaces de réflexion, car le cheminement spirituel est un travail de longue haleine et nécessite des haltes permettant de faire le point et d'intégrer ce que nous apprenons de nos rencontres avec autrui.

Le travail social n'a pas échappé à la mentalité de la concurrence, de la survie financière de l'organisme, de la performance. Les intervenantes et les intervenants sociaux ont une charge de plus en plus lourde de travail et sont souvent piégés dans les exigences administratives. Les réunions d'équipe sont souvent des mises au point techniques. Il n'y a plus de temps pour des réflexions plus approfondies. Il est à se demander si le taux élevé d'épuisement professionnel n'a pas un lien quelconque avec le fait que la suractivité a éteint en cours de route la quête spirituelle. Sommesnous devenus des voyageurs sans boussole, perdus dans l'immensité 
"La quête spirituelle ne saurait se contenter de réponses. Son dynamisme se trouve dans le questionnement. » des stimulations extérieures? Une démarche spirituelle nous permet de réviser nos objectifs de vie et nous aide à rechoisir l'essentiel et à lâcher prise sur tout ce qui est secondaire. Il est intéressant de constater que, récemment, certains organismes ont pris la décision de réserver dans leurs locaux un espace de silence et de réflexion destiné aux intervenants et aux usagers de services.

L'aventure spirituelle implique une ouverture afin de se laisser interpeller par l'autre. Elle nous garde constamment en état de questionnement. La quête spirituelle ne saurait se contenter de réponses. Son dynamisme se trouve dans le questionnement. C'est donc dire que dans le domaine du travail social, l'autre que nous aidons vient stimuler notre questionnement sur le sens de l'existence parce que l'expérience qu'il partage nous ouvre à de nouvelles dimensions. Dans cette perspective, on élimine la relation hiérarchique d'aidant-aidé pour y substituer une structure de dialogue dans laquelle l'un et l'autre contribuent mutuellement à l'apprentissage sur le sens de la vie.

\section{L'utilisation de la spiritualité dans la relation d'aide}

"C'est en dialoguant avec la personne sur sa spiritualité qu'elle nous livrera quelques indices sur le cadre qu'elle a développé en réponse aux situations dans une perspective plus large de l'existence."
De nombreuses ressources et de nombreux outils sont maintenant accessibles; prendre connaissance de quelques-unes de ces ressources peut certes aider l'intervenante ou l'intervenant dans sa propre vie et dans son travail d'intervention. S'exposer à d'autres courants spirituels peut nous éveiller à d'autres dimensions de la réalité, entre autres, assister à une conférence d'un moine bouddhiste, visiter un centre autochtone ou prendre rendez-vous avec un Ancien, lire un livre sur le judaïsme ou, dans un milieu de travail où la confiance règne dans une équipe de travail, organiser pendant l'heure du dîner un café-rencontre, portant sur le thème : "la spiritualité pour moi, c'est...".

Contrairement aux autres modèles d'intervention en travail social où intervenantes et intervenants ont appris à appliquer des techniques spécifiques de diverses problématiques, la spiritualité n'a pas de cadre technique à appliquer en tant que tel. C'est en 
dialoguant avec la personne sur sa spiritualité qu'elle nous livrera quelques indices sur le cadre qu'elle a développé en réponse aux situations dans une perspective plus large de l'existence.

Pour aider l'intervenante ou l'intervenant à se sentir à l'aise de poser des questions rattachées au domaine spirituel, tant dans sa propre vie que dans celle de ses clients et clientes, quatre éléments communs à la plupart des religions peuvent servir de point de départ :

- Le constat de la souffrance dans le monde;

- L'égoïsme humain qui est souvent la cause de cette souffrance;

- Une force de vie, plus grande que l'ego humain. Cette force peut être appelée Dieu, le grand esprit, l'Univers, Bouddha, la Nature, la grande puissance, etc.

- Les pratiques spirituelles telles que la prière, la lecture de textes sacrés, la méditation ou les œuvres caritatives qui peuvent nous connecter avec cette force supérieure et réduire la souffrance humaine.

En se basant sur ces quatre éléments, l'intervenante ou l'intervenant peut poser des questions ouvertes à la personne afin de mieux comprendre sa spiritualité et où elle se situe dans cet ensemble de réflexions, par exemple, "Qu'est-ce qui vous a aidée à surmonter la dernière crise? Comment cela vous a-t-il aidée? Qu'avez-vous appris au terme de cette situation?"

Pour les intervenants et les intervenantes qui se sentent plus à l'aise de travailler dans une structure établie, les éléments suivants peuvent être pris en considération pendant l'intervention :

- L'affiliation spirituelle

- Les croyances

- Les pratiques spirituelles

- L'apport de la spiritualité dans la vie de la personne

- Les valeurs spirituelles

- Les expériences spirituelles

- Le cheminement spirituel

- La conception du bien-être spirituel 
"Certaines cultures, je pense entre autres aux cultures autochtones, ont cependant une longue tradition dans cette approche holistique. Nous aurions avantage à puiser dans la richesse de leur expérience, non pour copier, mais pour apprendre. "
Il importe que cette cueillette d'information soit faite dans un climat de conversation avec la personne afin qu'elle se sente libre de répondre comme elle l'entend, et non à partir de questions dirigées qui l'astreindraient à répondre selon ce que l'intervenante ou l'intervenant veut entendre.

Pour conclure, de nombreux débats restent à prévoir sur la place de la spiritualité dans le travail social. Cela nous amène à examiner nos pratiques d'intervention. Certaines cultures, je pense entre autres aux cultures autochtones, ont cependant une longue tradition dans cette approche holistique. Nous aurions avantage à puiser dans la richesse de leur expérience, non pour copier, mais pour apprendre.

Nous mentionnions ci-dessus que la fermeture à tout ce qui est perçu comme étranger, à l'inconnu, constitue un des grands drames humains. Or, l'« étranger " qui peut représenter les plus grands défis est la vie elle-même, avec ses possibilités toujours nouvelles et cette crainte qu'elle inspire parfois devant son immense potentiel. Il peut être plus sécurisant de nous satisfaire de ce que nous avons et de ce que nous sommes, parce que nous sommes en terrain connu. Toutefois, nous pouvons prendre le risque d'aller plus loin en nous aventurant dans l'exploration d'alternatives. Reste qu'il faut plonger avec le discernement d'un regard critique.

\section{Bibliographie}

COLLECTIF CLIO (1992). L’histoire des femmes au Québec depuis quatre siècles, Québec, Québec Loisirs.

COUNCIL ON SOCIAL WORK EDUCATION (s.d.). "Integrating Spirituality into Social Work Practice:The Reflections on Spirituality and Aging (ROSA) Model”, Paper, Saint Louis University, School of Social Service.

DEFIORES, S. (1987). "Spiritualité contemporaine ", dans Dictionnaire de la vie spirituelle, Paris, Cerf, 1061-1078.

MOORE, T. (2000). Original Self, New York, Harper Collins.

OCHS, C. (1983). Women and Spirituality, Totowa, Rowman \& Allanheld.

WEBER, C.L. (1987). WomanChrist, San Francisco, Harper \& Row.

ZAPF, M.K. (2003). "Exploring the Spiritual Dimensions of Person and Place", Paper Presentation for Alberta College of Social Workers Annual Conference 2003, Edmonton. 
ZAPPONE, K.E. (1996). “The Faith of Feminists: Charting New Territory”, dans Vox Feminarum, vol. 1 no. $1,5-18$.

\section{Notes}

1 J.Moltmann (1974). L'homme. Essai d'anthropologie chrétienne, (traduit de l'allemand), Cerf-Mame, 12.

2 A. Manaranche (1972). Dieu vivant et vrai, Paris, Seuil, 67-68. 